\title{
LOCALIZATION OF MOBILE EQUIPMENT IN RADIO ENVIRONMENTS WITH NO LINE-OF-SIGHT PATH
}

\section{Jie Chen, Feng Jiang, A. Lee Swindlehurst}

\author{
Center for Pervasive Communications and Computing \\ Dept. of EECS, University of California, Irvine \\ Irvine, CA 92697, USA \\ \{jie.chen, feng.jiang, swindle\}@uci.edu
}

\author{
Jóse A. López-Salcedo \\ Dept. of Telecommunications and Systems Engineering \\ Universitat Autònoma de Barcelona (UAB) \\ 08193 Bellaterra (Barcelona), Spain \\ jose.salcedo@uab.es
}

\begin{abstract}
In recent years, radio positioning has received increasing attention and found many applications in various areas. However, the existence of non-line-of-sight (NLOS) paths introduces considerable positioning errors. In this paper, we propose a two-step approach in order to deal with pure NLOS scenarios based on a simple assumption regarding the propagation environment. A nonlinear least squares (NLS) method is proposed for the initial estimation, followed by a Kalman filter-based method to track subsequent movements. Compared with previous studies, fewer measurements are required to be made. Simulation results are provided to show the performance of both methods.
\end{abstract}

Index Terms - Localization, positioning, non-line-ofsight propagation.

\section{INTRODUCTION}

In recent years, radio positioning has received increasing attention and found many applications in industrial, medical, public safety, and entertainment areas. The most successful positioning technology is the global positioning system, which is a medium-earth orbit satellite-based navigation system that provides location and timing information. However, it requires that there exist unobstructed line-of-sight (LOS) paths from satellites to reception devices. In many cases, radio signals are obstructed by physical obstacles like trees, buildings, and mountains, and only non-line-of-sight (NLOS) paths can be observed. Similar problems also arise for positioning systems based on signals from base stations (BS) in cellular systems, where the transmitted waves suffer various radiation phenomena such as diffraction, refraction, reflection and scattering before they arrive at the mobile station (MS). Similarly, complicated radio propagation occurs in densely populated urban areas due to the existence of man-made obstacles. For a moving car or person, simply turning at a corner

This work was supported by the California-Catalonia Engineering Innovation Program. into a side street may radically change the wireless environment and the moving object may lose its LOS path to the BS.

With the popularity of mobile communications, many localization schemes have been proposed, and most of them assume that a LOS propagation path exists between the transmitters and receivers. With this assumption in mind, the geometric relationship between the target device, whose position needs to be precisely estimated, and its reference points are exploited to obtain knowledge of the physical distances between them. The most commonly used measurements are the time-of-arrival (TOA) [1, 2], the time-difference-of-arrival (TDOA) [3], and the received signal strength (RSS) [4]. Even in cases where a LOS path exists, additional NLOS arrivals are almost unavoidable, and these NLOS signals act as the major source of interference that lowers the reliability of TOA and TDOA measurements, resulting in considerable positioning errors. Thus far, most research efforts on combating the effects of NLOS signals have focused on NLOS error mitigation, i.e., how to detect multipaths that could be mistakenly perceived as LOS paths and then removing their impact [5]. A pure NLOS environment poses a much more challenging task to overcome. In this case, it is impossible to make conventional TOA/TDOA measurements. A potential way to combat this difficulty is so-called location fingerprinting [6], which requires a dense deployment of reference devices.

In this paper, we exploit a single-bounce assumption on the propagation environment to deal with localization in pure NLOS environments where no direct LOS exists, and we provide a method based on nonlinear least squares (NLS) combined with the extended Kalman filter (EKF). A similar scenario was considered in [7], though the method of [7] requires additional measurements such as the angle-of-arrival (AOA) at the MS, which is of ten infeasible to obtain in the absence of a significant array aperture and a stationary reference orientation for the device. The method presented in our work only assumes time and angle measurements made at multiple BS.

The rest of the paper is organized as follows. In Section 2, we describe the system model and formulate the measurement equations. Section 3 proposes a method to estimate the initial 
MS position based on NLS, and then Section 4 tackles the problem of tracking subsequent changes in the MS position using the EKF. Simulation results are presented in Section 5, and Section 6 summarizes the paper.

\section{SYSTEM MODEL}

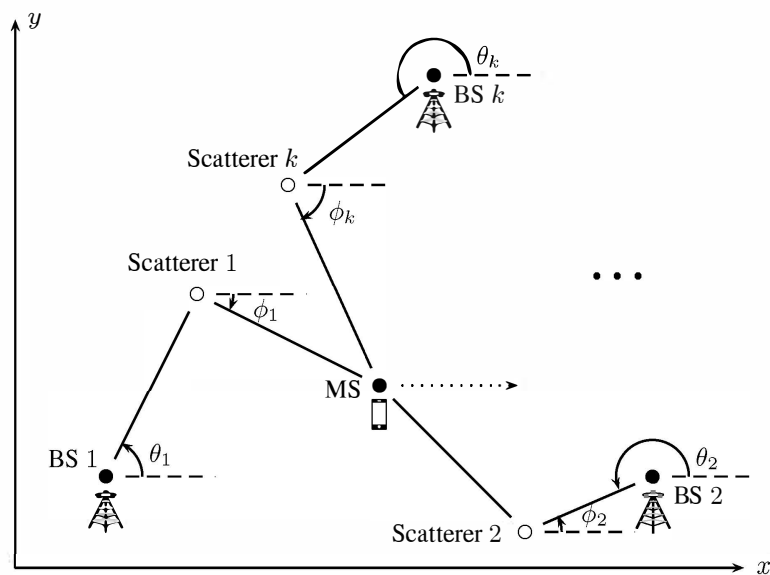

Fig. 1. A localization scenario with $k$ BS, each associated with one scatterer relative to the MS.

In this work, we consider a $k$-BS cellular system that observes uplink signals from a single MS, as depicted in Fig. 1. The location of all BS are known a priori. While the MS uplink signal may arrive at each BS via many multipaths, we assume that the path with the shortest delay is due to a singlebounce from a scatterer in a fixed but unknown location, and we assume that the TOA and AOA of this path at the BS can be determined. Note that in general, this scatterer is different for each BS. The TOA and AOA measurements at each BS are then forwarded to a centralized controller where the MS location estimate is obtained. We assume only signals from the scatterers can be observed at the BS, and no LOS path exists. As illustrated in Fig. $1,\left(x_{b s}^{(i)}, y_{b s}^{(i)}\right)$ and $\left(x_{s}^{(i)}, y_{s}^{(i)}\right)$ denote the coordinates of BS $i$ and the scatterer associated with BS $i$, respectively. The position of the MS is denoted by $(x(t), y(t))$, and $\left(x_{m s}, y_{m s}\right)=(x(0), y(0))$ is the initial position of MS.

Let $\theta_{i}$ be the AOA of the signal arriving at BS $i$ from scatterer $i$. Similarly, $\phi_{i}$ is the AOA of the signal path from the MS to scatterer $i$. We have the following relationships from simple geometry:

$$
\begin{gathered}
\tan \theta_{i}=\frac{y_{s}^{(i)}-y_{b s}^{(i)}}{x_{s}^{(i)}-x_{b s}^{(i)}} \\
\theta_{i}=\left\{\begin{aligned}
\arctan \frac{y_{s}^{(i)}-y_{b s}^{(i)}}{x_{s}^{(i)}-x_{b_{s}^{(i)}}^{(i)}} & \text { if } x_{s}^{(i)} \geq x_{b s}^{(i)} \\
\pi+\arctan \frac{y_{s}^{(i)}-y_{b s}^{(i)}}{x_{s}^{(i)}-x_{b s}^{(i)}} & \text { if } x_{s}^{(i)}<x_{b s}^{(i)}
\end{aligned}\right.
\end{gathered}
$$

$$
\begin{array}{r}
x_{s}^{(i)} \sin \theta_{i}-y_{s}^{(i)} \cos \theta_{i}=x_{b s}^{(i)} \sin \theta_{i}-y_{b s}^{(i)} \cos \theta_{i} \\
x_{s}^{(i)} \sin \phi_{i}-y_{s}^{(i)} \cos \phi_{i}=x_{m s} \sin \phi_{i}-y_{m s} \cos \phi_{i} .
\end{array}
$$

The delay measurements are given by

$$
\begin{aligned}
& \sqrt{\left(x_{s}^{(i)}-x(t)\right)^{2}+\left(y_{s}^{(i)}-y(t)\right)^{2}} \\
& +\sqrt{\left(x_{s}^{(i)}-x_{b s}^{(i)}\right)^{2}+\left(y_{s}^{(i)}-y_{b s}^{(i)}\right)^{2}}=c \tau_{i}(t),
\end{aligned}
$$

where $\tau_{i}(t)$ is the time delay from the MS to BS $i$ due to the associated scatterer.

We emphasize that only measurements of $\tau_{i}(t)$ and $\theta_{i}(t)$ are made at BS $i$. All other parameters, including $\phi_{i}(t)$, $\left(x_{s}^{(i)}, y_{s}^{(i)}\right)$, and $\left(x_{m s}, y_{m s}\right)$, are unknown and must be estimated, which adds significant difficulty to the localization problem.

\section{POSITIONING VIA NONLINEAR LEAST SQUARES}

We divide the localization process into two steps. First we assume that the MS moves at a constant velocity within a short period of time, so that we can formulate it as an NLS problem and solve it numerically. Based on the initial estimate obtained in the NLS step, we then use an extend Kalman filter to track the MS's movement, as discussed in the next section.

In this section, we discuss how the initial estimation can be done via NLS. The constant velocity assumption leads to

$$
\left.(x(t), y(t))\right|_{t=n \Delta t}=\left(x_{m s}+n \Delta t v_{x}, y_{m s}+n \Delta t v_{y}\right),
$$

where $\Delta t$ is the measurement interval, and $v_{x}$ and $v_{y}$ are the velocity components in the $x$ and $y$ directions. Hence the delay equation (5) can be rewritten as

$$
\begin{aligned}
& \sqrt{\left(x_{s}^{(i)}-\left(x_{m s}+n v_{x} \Delta t\right)\right)^{2}+\left(y_{s}^{(i)}-\left(y_{m s}+n v_{y} \Delta t\right)\right)^{2}} \\
& +\sqrt{\left(x_{s}^{(i)}-x_{b s}^{(i)}\right)^{2}+\left(y_{s}^{(i)}-y_{b s}^{(i)}\right)^{2}}=c \tau_{i}(n) .
\end{aligned}
$$

At time 0 , equation (7) becomes

$$
\begin{aligned}
& \sqrt{\left(x_{s}^{(i)}-x_{m s}\right)^{2}+\left(y_{s}^{(i)}-y_{m s}\right)^{2}} \\
& +\sqrt{\left(x_{s}^{(i)}-x_{b s}^{(i)}\right)^{2}+\left(y_{s}^{(i)}-y_{b s}^{(i)}\right)^{2}}=c \tau_{i}(0) .
\end{aligned}
$$

For all $n$ other than 0 , subtracting (8) from (7) leads to

$$
\begin{aligned}
& \sqrt{\left(x_{s}^{(i)}-\left(x_{m s}+n v_{x} \Delta t\right)\right)^{2}+\left(y_{s}^{(i)}-\left(y_{m s}+n v_{y} \Delta t\right)\right)^{2}} \\
& -\sqrt{\left(x_{s}^{(i)}-x_{m s}\right)^{2}+\left(y_{s}^{(i)}-y_{m s}\right)^{2}}=c \tau_{i}(n)-c \tau_{i}(0) .
\end{aligned}
$$




$$
\begin{aligned}
& \mathbf{P}_{i}=\left[\begin{array}{cc}
\sin \theta_{i} & -\cos \theta_{i} \\
\sin \phi_{i} & -\cos \phi_{i} \\
\sec \theta_{i}-\sec \phi_{i} & \csc \theta_{i}-\csc \phi_{i} \\
-v_{x} \Delta t+\frac{c}{2}\left(\tau_{i}(1)-\tau_{i}(0)\right) \sec \phi_{i} & -v_{y} \Delta t+\frac{c}{2}\left(\tau_{i}(1)-\tau_{i}(0)\right) \csc \phi_{i} \\
\vdots & \vdots \\
-N v_{x} \Delta t+\frac{c}{2}\left(\tau_{i}(N)-\tau_{i}(0)\right) \sec \phi_{i} & -N v_{y} \Delta t+\frac{c}{2}\left(\tau_{i}(N)-\tau_{i}(0)\right) \csc \phi_{i}
\end{array}\right] \\
& \mathbf{Q}_{i}=\left[\begin{array}{cc}
0 & 0 \\
-\sin \phi_{i} & \cos \phi_{i} \\
\sec \phi_{i} & \csc \phi_{i} \\
v_{x} \Delta t-\frac{c}{2}\left(\tau_{i}(1)-\tau_{i}(0)\right) \sec \phi_{i} & v_{y} \Delta t-\frac{c}{2}\left(\tau_{i}(1)-\tau_{i}(0)\right) \csc \phi_{i} \\
\vdots & \vdots \\
N v_{x} \Delta t-\frac{c}{2}\left(\tau_{i}(N)-\tau_{i}(0)\right) \sec \phi_{i} & N v_{y} \Delta t-\frac{c}{2}\left(\tau_{i}(N)-\tau_{i}(0)\right) \csc \phi_{i}
\end{array}\right] \\
& \mathbf{m}_{i}=\left[\begin{array}{c}
x_{b s}^{(i)} \sin \theta_{i}-y_{b s}^{(i)} \cos \theta_{i} \\
0 \\
2 c \tau_{i}(0)+x_{b s}^{(i)} \sec \theta_{i}+y_{b s}^{(i)} \csc \theta_{i} \\
\frac{c^{2}}{2}\left(\tau_{i}(1)-\tau_{i}(0)\right)^{2}-\frac{1}{2}\left(v_{x} \Delta t\right)^{2}-\frac{1}{2}\left(v_{y} \Delta t\right)^{2} \\
\vdots \\
\frac{c^{2}}{2}\left(\tau_{i}(N)-\tau_{i}(0)\right)^{2}-\frac{1}{2}\left(N v_{x} \Delta t\right)^{2}-\frac{1}{2}\left(N v_{y} \Delta t\right)^{2}
\end{array}\right]
\end{aligned}
$$

We then introduce $\phi_{i}$ into equation (9). After some mathematical manipulations, we get

$$
\begin{aligned}
& \frac{1}{2}\left(n v_{x} \Delta t\right)^{2}+\left(x_{m s}-x_{s}^{(i)}\right) n v_{x} \Delta t \\
& +\frac{1}{2}\left(n v_{y} \Delta t\right)^{2}+\left(y_{m s}-y_{s}^{(i)}\right) n v_{y} \Delta t \\
& -\frac{1}{2}\left(c \tau_{i}(n)-c \tau_{i}(0)\right)\left(x_{m s}-x_{s}^{(i)}\right) \sec \phi_{i} \\
& -\frac{1}{2}\left(c \tau_{i}(n)-c \tau_{i}(0)\right)\left(y_{m s}-y_{s}^{(i)}\right) \csc \phi_{i} \\
& =\frac{1}{2}\left(c \tau_{i}(n)-c \tau_{i}(0)\right)^{2} .
\end{aligned}
$$

When measurement noise is present, a NLS problem can be formulated based on (10) for all $i$ with $v_{x}, v_{y}, x_{m s}, y_{m s}, x_{s}^{(i)}$, $y_{s}^{(i)}$, and $\phi_{i}$ as adjustable variables. At first glance, it appears that the resulting NLS problem could be directly solved by numerical optimization methods. However, our experiments show that this problem has many local optima, and numerical approaches of ten result in large estimation errors. An alternative approach is described below to address this problem.

A further observation on (10) reveals that if the squared terms, $\left(n v_{x} \Delta t\right)^{2}$ and $\left(n v_{y} \Delta t\right)^{2}$, did not exist, the resulting NLS problem could be regarded as a separable problem [8]. So we move the squared terms to the right hand side of the equation, build up the NLS problem, and decompose the NLS problem into $\mathbf{H}(\boldsymbol{\alpha}) \boldsymbol{\beta}=\boldsymbol{\gamma}$, where $\boldsymbol{\alpha}=\left[v_{x}, v_{y}, \phi_{1}, \ldots, \phi_{k}\right]$ and $\boldsymbol{\beta}=\left[x_{s}^{(1)}, y_{s}^{(1)}, \ldots, x_{s}^{(k)}, y_{s}^{(k)}, x_{m s}, y_{m s}\right]$. We refer to this as a pseudo-separable NLS problem because separability in the strict sense requires $\gamma$ to only depend on the measurements, while in our case it also depends on the parameters to be estimated. Since the numerical methods used to solve the separable problem, e.g. the variable projection method [9], are iterative in nature, we can use estimates of $v_{x}$ and $v_{y}$ from the last iteration to evaluate the squared terms in $\gamma$ for the next iteration. Simulations show that this approximate algorithm converges and results in acceptable estimation performance.

Putting everything together, we have

$$
\left[\begin{array}{cccccc}
\mathbf{P}_{1} & 0 & \ldots & \ldots & 0 & \mathbf{Q}_{1} \\
\vdots & \ddots & \vdots & \vdots & \vdots & \vdots \\
0 & \ldots & \mathbf{P}_{i} & \ldots & 0 & \mathbf{Q}_{i} \\
\vdots & \vdots & \vdots & \ddots & \vdots & \vdots \\
0 & \cdots & \cdots & 0 & \mathbf{P}_{k} & \mathbf{Q}_{k}
\end{array}\right]\left[\begin{array}{c}
x_{s}^{(1)} \\
y_{s}^{(1)} \\
\vdots \\
x_{s}^{(k)} \\
y_{s}^{(k)} \\
x_{m s} \\
y_{m s}
\end{array}\right]=\left[\begin{array}{c}
\mathbf{m}_{1} \\
\vdots \\
\mathbf{m}_{i} \\
\vdots \\
\mathbf{m}_{k}
\end{array}\right]
$$

which is in the decomposed form of $\mathbf{H}(\boldsymbol{\alpha}) \boldsymbol{\beta}=\boldsymbol{\gamma}$. The definitions of $\mathbf{m}_{i}, \mathbf{P}_{i}$ and $\mathbf{Q}_{i}$ are on the top of this page, where $\mathbf{m}_{i} \in \mathbb{R}^{(N+3) \times 1}, \mathbf{P}_{i}$ and $\mathbf{Q}_{i} \in \mathbb{R}^{(N+3) \times 2}$. The total number of samples, $N$, is chosen offline before the positioning process starts.

\section{POSITIONING VIA EXTENDED KALMAN FILTER}

Once the initial estimations are obtained, we appeal to the Kalman filter to track subsequent movement of the MS, under which the constant velocity assumption is relaxed.

Let $\boldsymbol{\eta}(n)$ and $\mathbf{w}(n)$ be the transpose of

$$
\left[x(n) y(n) v_{x}(n) v_{y}(n) x_{s}^{(1)}(n) y_{s}^{(1)}(n) \cdots x_{s}^{(k)}(n) y_{s}^{(k)}(n)\right]
$$




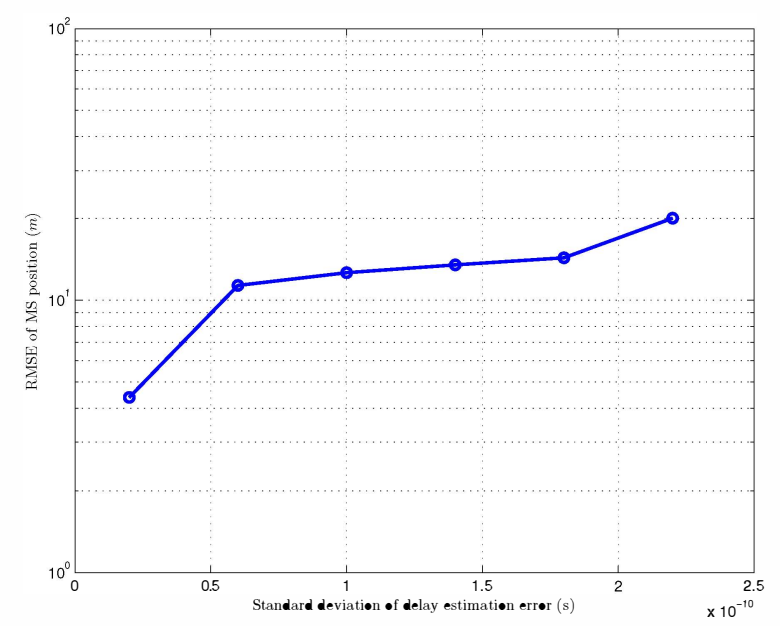

Fig. 2. RMSE of MS position

and

$\left[w_{x}(n) w_{y}(n) w_{v_{x}}(n) w_{v_{y}}(n) w_{x_{s}^{(1)}}(n) w_{y_{s}^{(1)}}(n) \cdots w_{x_{s}^{(k)}}(n) w_{y_{s}^{(k)}}(n)\right]$,

respectively. Also define $\boldsymbol{\mu}(n)=\left[\tau_{1}(n) \theta_{1}(n) \cdots \tau_{k}(n) \theta_{k}(n)\right]^{T}$ as the delay and AOA measurement vector, and $\mathbf{z}(n)=$ $\left[z_{\tau_{1}}(n) z_{\theta_{1}}(n) \cdots z_{\tau_{k}}(n) z_{\theta_{k}}(n)\right]^{T}$ as the measurement noise vector. Then the dynamic model of the Kalman filter is

$$
\boldsymbol{\eta}(n)=\left[\begin{array}{cc}
\mathbf{A} & \mathbf{0} \\
\mathbf{0} & \mathbf{I}_{2 k}
\end{array}\right] \boldsymbol{\eta}(n-1)+\mathbf{w}(n),
$$

where

$$
\mathbf{A}=\left[\begin{array}{cccc}
1 & 0 & \Delta t & 0 \\
0 & 1 & 0 & \Delta t \\
0 & 0 & 1 & 0 \\
0 & 0 & 0 & 1
\end{array}\right]
$$

and the observation model is $\boldsymbol{\mu}(n)=\mathbf{h}(\boldsymbol{\eta}(n))+\mathbf{z}(n)$, where $\mathbf{h}$ is a stack of equations (2) and (5). Due to the nonlinear nature of $\mathbf{h}$, we use the EKF [8] to do the tracking.

\section{SIMULATIONS}

Assume three BS located at $(10,10),(120,20)$, and $(80,80)$ in units of meters, with associated scatterers at $(22,5)$, $(108,15)$, and $(90,68)$, respectively. The initial position of the MS is $(50,30)$, and during the NLS stage, the MS moves northbound at the speed of $3 \mathrm{~km} / \mathrm{hr}$ for 5 meters. Except for the coordinates of the BS, all other parameters are unknown a priori and need to be estimated. Figs. 2-3 show the RMS error of the MS position and velocity obtained by the NLS algorithm as a function of the standard deviation of the delay estimation error at each BS. The standard deviation of the AOA estimation error is assumed to be $1^{\circ}$. Fig. 4 shows the performance of the EKF tracker once the initial MS position

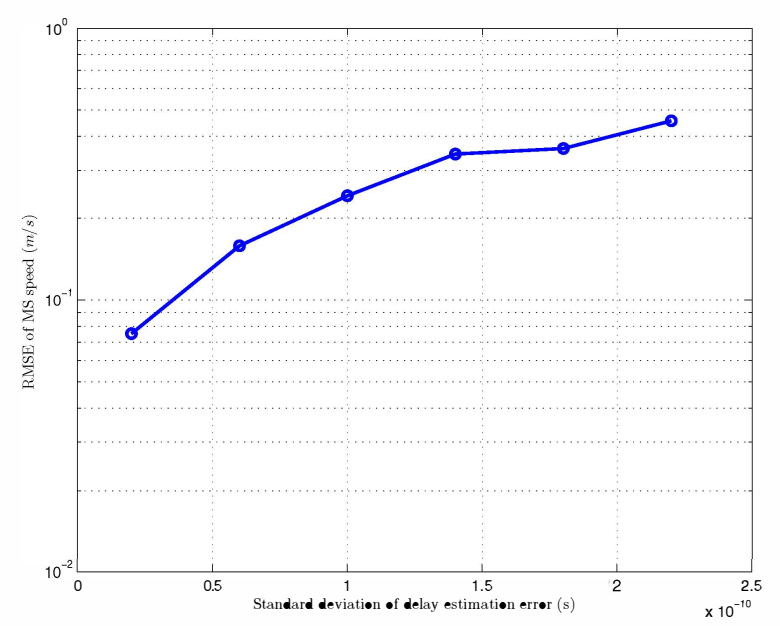

Fig. 3. RMSE of MS speed

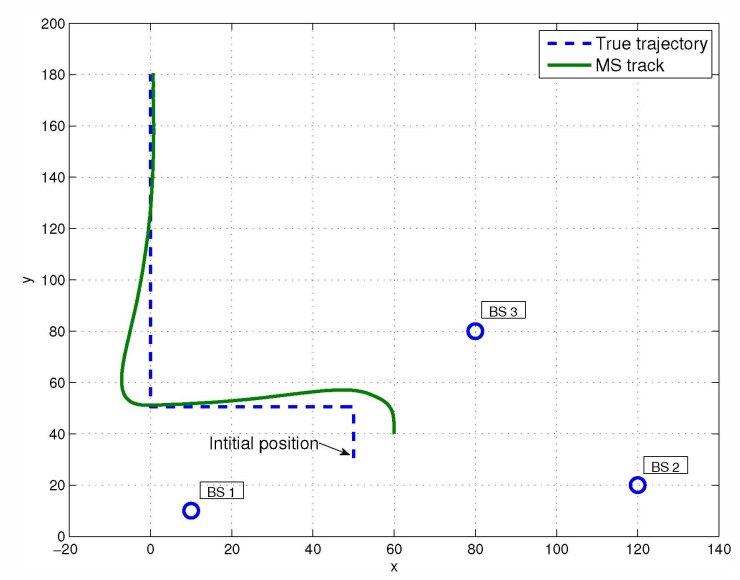

Fig. 4. True and estimated MS trajectory

has been estimated using NLS, assuming a standard deviation on the delay error of $20 \mathrm{ps}$. The initial estimate is in error by around $15 \mathrm{~m}$, but we see that the EKF is able to reduce the error below $1 \mathrm{~m}$ during periods of straight-line motion.

\section{CONCLUSION}

In this paper, we have proposed a new method for localizing a mobile user in a pure NLOS environment using several BS. An NLS method is proposed for the initial estimation, followed by a EKF to track the subsequent movements of MS. Compared to previous studies, only uplink measurements made by the BS are required to localize the MS. Simulation results are provided to show the localization performance of the methods. Possible areas of future work include use of weighted or total LS to improve the accuracy of the initial location estimate, and application of the idea to more complicated environments with multiple scatterers per BS. 


\section{REFERENCES}

[1] K.W. Cheung, H.C. So, W.-K. Ma, and Y.T. Chan, "Least squares algorithms for time-of-arrival-based mobile location," Signal Processing, IEEE Transactions on, vol. 52, no. 4, pp. 1121 - 1130, April 2004.

[2] Xin Wang, Zongxin Wang, and B. O'Dea, “A TOA-based location algorithm reducing the errors due to non-line-ofsight (NLOS) propagation," Vehicular Technology, IEEE Transactions on, vol. 52, no. 1, pp. 112 - 116, Jan. 2003.

[3] Y.T. Chan and K.C. Ho, "A simple and efficient estimator for hyperbolic location," Signal Processing, IEEE Transactions on, vol. 42, no. 8, pp. 1905 -1915, Aug. 1994.

[4] N. Patwari, A.O. Hero III, M. Perkins, N.S. Correal, and R.J. O'Dea, "Relative location estimation in wireless sensor networks," Signal Processing, IEEE Transactions on, vol. 51, no. 8, pp. 2137 - 2148, Aug. 2003.

[5] Kaveh Pahlavan, Ferit O. Akgul, Mohammad Heidari, Ahmad Hatami, John M. Elwell, and Robert D. Tingley, "Indoor geolocation in the absence of direct path," Wireless Communications, IEEE, vol. 13, no. 6, pp. 50 -58, Dec. 2006.

[6] Jeffrey Hightower and Gaetano Borriello, "Location systems for ubiquitous computing," Computer, vol. 34, no. 8, pp. 57-66, Aug. 2001.

[7] Chee Kiat Seow and Soon Yim Tan, "Non-line-of-sight localization in multipath environments," IEEE Trans. Mobile Comput., vol. 7, no. 5, pp. 647-660, 2008.

[8] S.M. Kay, Fundamentals Of Statistical Signal Processing, Prentice Hall, 2001.

[9] G. H. Golub and V. Pereyra, "The differentiation of pseudo-inverses and nonlinear least squares problems whose variables separate," SIAM Journal on Numerical Analysis, vol. 10, no. 2, pp. 413-432, 1973. 